\title{
Phase II TPDCV protocol for pediatric low-grade hypothalamic/chiasmatic gliomas: 15-year update
}

\author{
Kavita K. Mishra $\cdot$ Sarah Squire $\cdot$ Kathleen Lamborn • \\ Anuradha Banerjee $\cdot$ Nalin Gupta $\cdot$ William M. Wara $\cdot$ \\ Michael D. Prados • Mitchel S. Berger · Daphne A. Haas-Kogan
}

Received: 9 December 2009/ Accepted: 21 February 2010/Published online: 11 March 2010

(C) The Author(s) 2010. This article is published with open access at Springerlink.com

\begin{abstract}
To report long-term results for children with low-grade hypothalamic/chiasmatic gliomas treated on a phase II chemotherapy protocol. Between 1984 and 1992, 33 children with hypothalamic/chiasmatic LGGs received TPDCV chemotherapy on a phase II prospective trial. Median age was 3.0 years (range 0.3-16.2). Twelve patients (36\%) underwent STRs, $14(42 \%)$ biopsy only, and seven (21\%) no surgery. Twenty patients $(61 \%)$ had pathologic JPAs, nine (27\%) grade II gliomas, and four (12\%) no surgical sampling. Median f/u for surviving patients was 15.2 years (range 5.3-20.7); 20 of the 23 surviving patients had 14 or more years of follow-up. Fifteen-year PFS and OS were 23.4 and $71.2 \%$, respectively. Twenty-five patients progressed, of whom 13 are NED, two are AWD, and 10 have died. All children who died were diagnosed and first treated at age three or younger. Age at diagnosis was significantly associated with
\end{abstract}

Presented at the American Society for Therapeutic Radiology and Oncology, Los Angeles, CA, October 30, 2007.

K. K. Mishra · S. Squire · W. M. Wara ·

D. A. Haas-Kogan $(\bowtie)$

Department of Radiation Oncology, University of California,

San Francisco, 1600 Divisadero, San Francisco, CA 94143, USA

e-mail: dhaaskogan@radonc.ucsf.edu

K. Lamborn · A. Banerjee · N. Gupta · M. D. Prados ·

M. S. Berger · D. A. Haas-Kogan

Department of Neurosurgery, Brain Tumor Research Center,

University of California, San Francisco, San Francisco,

CA, USA

relapse and survival $(P=0.004$ for PFS and $P=0.037$ for OS). No PFS or OS benefit was seen with STR versus biopsy/no sampling ( $P=0.58$ for PFS, $P=0.59$ for OS). For patients with JPAs and WHO grade II tumors, the 15year PFS was 18.8 and $22.2 \%(P=0.95)$ and 15 -year OS was 73.7 and $55.6 \%(P=0.17)$, respectively. Upfront TPDCV for children with hypothalamic/chiasmatic LGGs resulted in 15-year OS of $71.2 \%$ and 15-year PFS of $23.4 \%$. No survival benefit is demonstrated for greater extent of resection. Age is a significant prognostic factor for progression and survival.

Keywords Low-grade gliomas - Pediatric .

Chemotherapy $\cdot$ Radiation $\cdot$ Hypothalamic/chiasmatic

$\begin{array}{ll}\text { Abbreviations } \\ \text { WHO } & \text { World Health Organization } \\ \text { LGG } & \text { Low-grade glioma } \\ \text { PFS } & \text { Progression-free survival } \\ \text { OS } & \text { Overall survival } \\ \text { GTR } & \text { Gross total resection } \\ \text { STR } & \text { Subtotal resection } \\ \text { UCSF } & \text { University of California San Francisco } \\ \text { RT } & \text { Radiation therapy } \\ \text { NED } & \text { No evidence of disease } \\ \text { AWD } & \text { Alive with disease } \\ \text { HR } & \text { Hazard ratio } \\ \text { JPA } & \text { Juvenile pilocytic astrocytoma } \\ \text { OPG } & \text { Optic pathway glioma } \\ \text { BTRC } & \text { Brain Tumor Research Center } \\ \text { KPS } & \text { Karnofsky Performance Score } \\ \text { COG } & \text { Children's Oncology Group } \\ \text { VEGF } & \text { Vascular endothelial growth factor receptor } \\ \text { MAPK } & \text { Mitogen-activated protein kinase }\end{array}$




\section{Introduction}

The treatment of hypothalamic/chiasmatic LGGs represents a unique clinical challenge because involvement of eloquent midline structures frequently precludes extensive resection of these tumors. Radiation can effectively reduce tumor bulk and slow or halt progression of pediatric midline LGGs; however it can also lead to long-term complications including neurocognitive deficits, hormonal changes, growth delay, and radionecrosis [1, 2]. In an effort to defer radiation, upfront chemotherapy has been studied for the treatment of hypothalamic/chiasmatic LGGs [2, 3]. Although overall survival (OS) rates for pediatric optic pathway gliomas are excellent (5-year OS of $80-90 \%)$, the majority of children (60-70\%) initially managed with chemotherapy will experience disease progression [4].

Due to limited number of cases, differences in treatment regimens, dearth of randomized studies, and often inadequate follow up, a lack of consensus persists regarding the optimal treatment of pediatric hypothalamic/chiasmatic LGGs. In 1984, we instituted a study protocol at the University of California San Francisco-Brain Tumor Research Center (UCSF-BTRC) to evaluate the efficacy of upfront chemotherapy alone for treatment of hypothalamic/ chiasmatic LGGs in our pediatric population. BTRC protocol 8422 was a planned phase II prospective trial evaluating TPDCV chemotherapy in children age 18 and younger. TPDCV is a five-drug regimen consisting of 6thioguanine, procarbazine, dibromodulcitol, 1-(2-chloroethyl)-3-cyclohexyl-1-nitrosourea (CCNU), and vincristine and described in detail in the original publication $[5,6]$. This report updates the follow-up on the subset of pediatric patients with hypothalamic/chiasmatic LGGs who were treated with an outpatient upfront TPDCV chemotherapy regimen alone and no radiation at the time of initial treatment.

\section{Materials and methods}

Between March 9, 1984 and January 29, 1992, 33 children ages 18 and younger with low-grade (WHO grade I and II) hypothalamic/chiasmatic gliomas were enrolled on UCSFBTRC protocol 8422 , a single arm phase II prospective trial. On this protocol, patients received outpatient TPDCV chemotherapy as their primary treatment (details of the protocol have been previously published, see [5, 6]). All patient characteristics, treatment parameters, and survival data were extracted from clinical records, radiographic information, and active follow-up obtained from the Departments of Radiation Oncology, Neuro-oncology, and/ or Neurosurgery at UCSF. Informed consent was obtained from parents or guardians of each child before entry into the trial. This study was performed after approval by local Human Investigations Committees.

Subjects' original pathological diagnoses from UCSF neuropathology were used for classification. Tumor types included low-grade astrocytoma, oligodendroglioma, and mixed oligoastrocytoma. Histopathologic confirmation of the diagnosis was required in all patients except those with diffusely infiltrating diencephalic lesions who had neurofibromatosis and those with optic chiasm lesions extending posteriorly along the optic radiations or causing enlargement of the optic nerves.

Extent of resection was based on pre- and post-operative radiographic imaging studies as well as operative reports. There were no gross total resections (GTRs) in the study population. Subtotal resection (STR) included those with bulk tumor resection and residual tumor on radiographic imaging; biopsy included those with surgical tumor sampling only for sole purpose of histologic diagnosis.

Chemotherapy details are outlined in previous reports $[5,6]$. Briefly, a nitrosourea based multi-agent chemotherapy was given as follows. TPDCV chemotherapy cycles consisted of 6-thioguanine (hour 0-66), procarbazine (hour 60-78), and dibromodulcitol (hour 60) given before lomustine (CCNU; hour 72); vincristine was then given after CCNU (day 14 and 28). Dosing was as follows: 6-thioguanine $30 \mathrm{mg} / \mathrm{m}^{2}$ orally every $6 \mathrm{~h} \times 12$ doses, procarbazine $50 \mathrm{mg} / \mathrm{m}^{2}$ orally every $6 \mathrm{~h} \times 4$ doses, dibromodulcitol $400 \mathrm{mg} / \mathrm{m}^{2}$, CCNU $110 \mathrm{mg} / \mathrm{m}^{2}$, vincristine $1.4 \mathrm{mg} / \mathrm{m}^{2}$ IV push. Each cycle was planned at 42-day intervals. Patients were treated for six treatment cycles or until tumor progressed, whichever came first. Chemotherapy was modified based on hematologic and other agentspecific toxicities. Dose escalations were not allowed.

No child received up-front radiation as part of his/her primary treatment. Patients with prior radiation or chemotherapy were excluded. The decision of whether to administer radiation or other treatment modalities as salvage therapy was made at the discretion of the treating physician.

During the follow-up period, information regarding clinical status, radiographic status, salvage therapies, progression, and survival were noted. Patients underwent contrast-enhanced MRI or CT imaging within 2 weeks before study entry and thereafter every 6 weeks during treatment. During the follow-up period, stable and responding patients underwent MR or CT imaging every 3 months for the first year, every 4 months the second year, every 6 months the third year, then annually, and if stable had scans at 1-3 year intervals thereafter.

PFS and OS rates were calculated from date of diagnosis. Life-table methods were used to estimate PFS and OS [7]. Five- and 10-year PFS and OS estimates were calculated using the Kaplan-Meier method, with standard 
errors calculated by Greenwood's formula. Elapsed time from diagnosis to an event or last follow-up was used to compute PFS and OS probabilities. In analyses of PFS, last follow-up dates for patients not experiencing an event were based on most recent clinical and/or radiographic followup that delineated disease status. For OS, deaths, regardless of cause, were coded as events. For PFS, an event was defined as relapse, progression or death during the period of active follow-up. To assess variables influencing PFS and OS, univariate analyses using log-rank tests and proportional hazards models were performed.

\section{Results}

Demographics

Thirty-three patients were enrolled in UCSF-BTRC phase II protocol 8422 between 1984 and 1992. Median age at diagnosis was 3.0 years (range $0.3-16.2$ years). The ratio of female to male was 20:13 (61:39\%); six patients (18\%) were diagnosed with neurofibromatosis. Tumor location was classified as hypothalamic or chiasmatic in 27 and 6 patients (82 and 18\%), respectively. The median Karnofsky Performance Score (KPS) at diagnosis was 80 (range 70100). No patient had a GTR, 12 patients (36\%) underwent STR, and $14(42 \%)$ had biopsy at the time of diagnosis. Seven patients $(21 \%)$ had no surgery at time of diagnosis, of which three had subsequent surgery at the time of recurrence. Twenty patients (61\%) had pathologic JPAs, 9 patients (27\%) had grade II gliomas, and 4 patients (12\%) had no surgical sampling. Table 1 summarizes the clinical characteristics for the entire cohort.

\section{Follow Up}

Median follow-up for surviving patients was 15.2 years (range 5.3-20.7 years). All but three of the surviving patients had 14 or more years of follow-up with regular interval radiographic scans. All surviving patients had at least 5 years of follow-up. Two patients had clinical follow-up of NED status however their most recent MRI/CT scan was dated more than 3 years prior to date of last clinical follow-up. Three patients were lost to follow-up after 5.3, 6.5, and 9.3 years, respectively, of follow-up. Of these three patients, the first had progressed at 3.9 years but salvage therapy was given elsewhere and no further records could be located.

\section{Overall PFS and OS}

Median PFS was 3.1 years, median OS has not been reached. Five-year PFS and OS were $30.3 \pm 8.0$ and
Table 1 Patient characteristics and treatment details

\begin{tabular}{ll}
\hline Variables & Total $(N=33)$ \\
\hline Age-median (range), years & $3.0(0.3-16.2)$ \\
Follow-up-median (range) ${ }^{\mathrm{a}}$, years & $15.2(5.3-20.7)$ \\
Pts with $\geq 5$ years of follow-up/total surviving pts & $23 / 23(100 \%)$ \\
Gender-Female:male (\%) & $20: 13(61: 39 \%)$ \\
Tumor location-hypothalamic: chiasmatic & $27: 6(82: 18 \%)$ \\
Neurofibromatosis & $6(18 \%)$ \\
KPS, median (range) & $80(70-100)$ \\
Histology: JPA & $20(61 \%)$ \\
Grade II LGG & $9(27 \%)$ \\
No histologic sampling & $4(12 \%)$ \\
Extent of resection at diagnosis & \\
Gross total resection & 0 \\
Subtotal resection & $12(36 \%)$ \\
Biopsy only & $14(42 \%)$ \\
No surgery & $7(21 \%)$ \\
\hline
\end{tabular}

${ }^{a}$ Follow-up for surviving patients

b Three of these 7 patients had surgery at time of progression

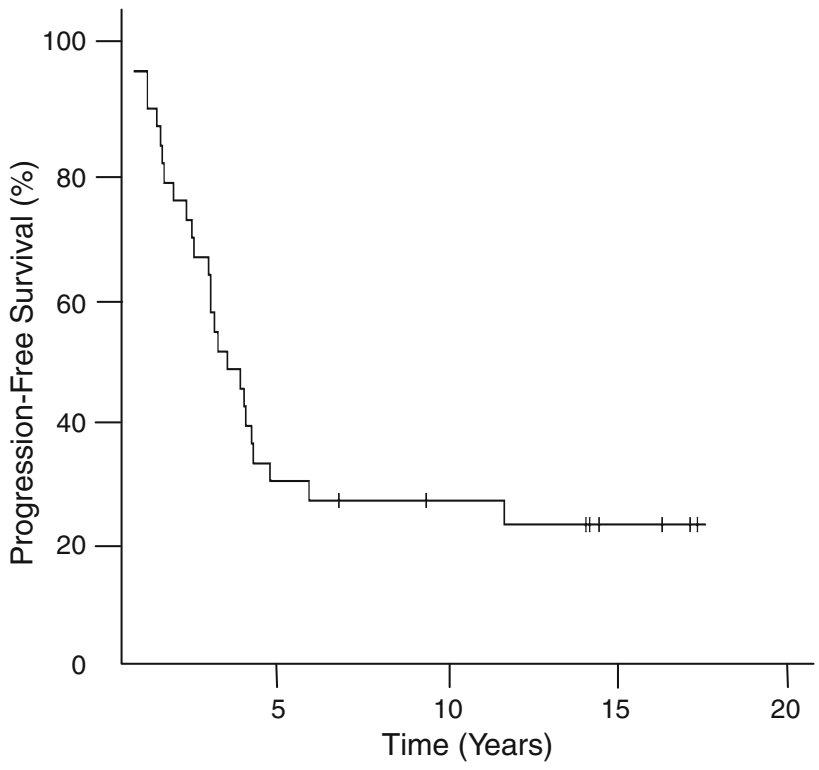

Fig. 1 Progression-free survival of 33 patients on phase II TPDCV chemotherapy trial for pediatric low-grade hypothalamic/chiasmatic gliomas

$90.9 \pm 5.0 \%$, respectively. Fifteen-year PFS and OS were $23.4 \pm 7.6$ and $71.2 \pm 8.1 \%$, respectively. Figures 1 and 2 show the Kaplan-Meier curves for PFS and OS, respectively. Twenty-five patients progressed during the follow-up period and underwent subsequent radiation, chemotherapy, and/or surgical salvage. All but one progression event occurred within six years of diagnosis. For the patients that died, median PFS was 1.9 years (range 0.6-5.7 years) and median OS was 6.6 years (range 2.1-15.4 years). Of the 11 patients 


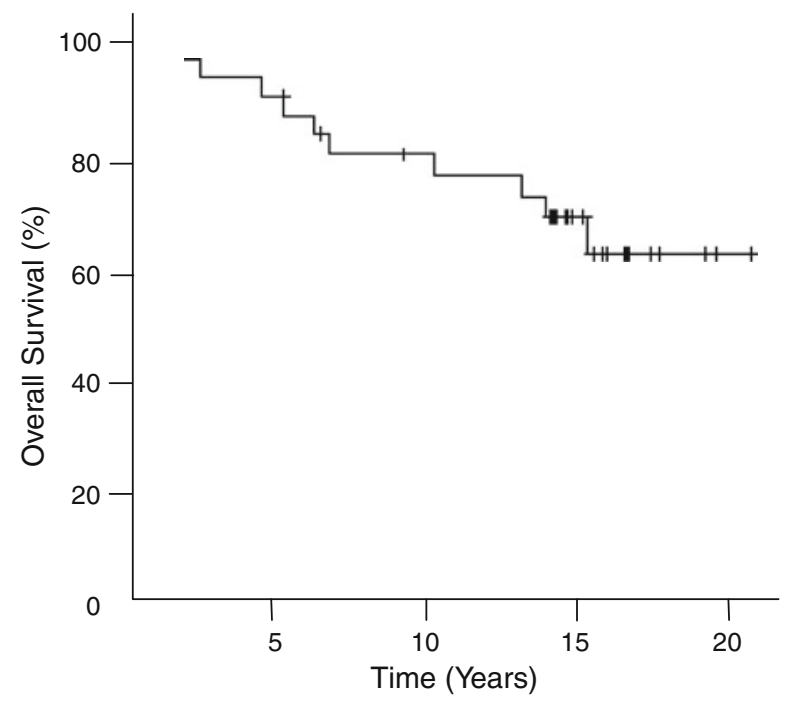

Fig. 2 Overall survival of 33 patients on phase II TPDCV chemotherapy trial for pediatric low-grade hypothalamic/chiasmatic gliomas

who had surgery as part of their salvage therapy, seven recurred as low grade gliomas (six JPAs and one low grade astrocytoma). For the four others, pathology was not available. All children who have died were diagnosed and first treated at age 3 or younger.

Given the use of an alkylator-based regimen in this study, we specifically inquired about the development of secondary malignancies. One patient developed a second primary many years after therapy, an osteosarcoma that arose within the radiation field. This patient was a Caucasian female with a grade II hypothalamic tumor diagnosed at age 2.3 years; she did not have neurofibromatosis. She had received chemotherapy upfront according to protocol guidelines and then radiation to $54 \mathrm{~Gy}$ for first salvage and procarbazine for second progression. She died due to progression of her secondary sarcoma 15.4 years after her primary diagnosis.

\section{Salvage therapy}

Twenty-five patients progressed during the period of active follow-up. Of these 25, 13 are salvaged and currently NED with a median follow-up of 15.5 years. Two are alive with disease, and 10 are dead at last follow-up. Of the 25 patients who progressed, 24 had their first progression event within 6 years of diagnosis. Salvage therapy at time of first progression included radiation (RT; $N=17$ ), chemotherapy $(N=8)$, and surgery $(N=11)$. Table 2 summarizes the salvage therapies utilized and number of patients currently NED according to salvage treatment modality. Of note, one patient who progressed during his TPDCV chemotherapy regimen was salvaged with single agent carboplatin and is currently NED with last follow-up at 15.2 years since diagnosis.
Table 2 Components of salvage therapy and no-evidence-of-disease (NED) status

\begin{tabular}{lll}
\hline Total salvage therapy & $\begin{array}{l}\text { Number } \\
\text { of pts }\end{array}$ & Number NED \\
\hline RT alone & 7 & 3 \\
Chemotherapy alone & 2 & 1 \\
Surgery alone & 2 & 2 \\
RT + chemo & 3 & 1 \\
RT + surgery & 6 & 3 \\
Chemotherapy + surgery & 2 & 1 \\
RT + chemotherapy + surgery & 1 & 0 \\
\hline
\end{tabular}

Seventeen of the 25 patients who progressed underwent subsequent radiation as part of their salvage therapy. The vast majority received a total dose of 50.4-54 Gy in 1.82 Gy fractions to the primary tumor site with margin. One patient received hyperfractionated RT (1.15 Gy BID to $63.4 \mathrm{~Gy}$ ) and two patients received stereotactic radiosurgery as part of their radiation course. A total of seven patients of the 17 undergoing subsequent RT were successfully salvaged and NED at last follow-up.

Prognostic factors

Age

Eighteen children enrolled on study were 3 years or younger at diagnosis ("young children") and 15 were older than 3 years at diagnosis ("old children"). A total of 10 young children died, 7 remained NED, and 1 was AWD at last follow-up. Age at diagnosis was significantly associated with OS $(P=0.037)$. All ten children that died in the entire study cohort were 3 years of age or younger at diagnosis. For the children that died, median PFS was 1.9 years (range 0.6-5.7) and median OS was 6.6 years (range 2.1-15.4).

Progression occurred more frequently and earlier in the $\leq 3$ year old group, with 17 of 18 progressing among young patients versus eight of 15 progressing among older children. Age at diagnosis was significantly associated with PFS $(P=0.004)$; with a median PFS of 2.6 versus 6.6 years for the young versus old group, respectively.

Of the 17 progressing young children, 13 underwent RT as part of their salvage therapy with eight dead at last follow-up; and four received chemotherapy and/or surgical salvage, of who two died. Of the 13 young children that underwent salvage RT, the average delay until RT was given from time of diagnosis was 2.7 years. For 10 of the 13 , radiation delivery was successfully delayed until after the age of 3. Three children underwent salvage RT before turning 3 years of age at age 2.9, 2.7, and 1.5 and at last follow-up are DOD, NED, and DOD, respectively. 


\section{Extent of surgical resection}

Overall, no PFS or OS benefit was seen with STR versus biopsy/no sampling ( $P=0.58$ for PFS, $P=0.59$ for OS). During the follow-up period, progression occurred in 16 of $21(76 \%)$ patients with biopsy or no surgical sampling and nine of $12(75 \%)$ patients with STRs. Death occurred in three of $21(33 \%)$ patients with biopsy or no surgical sampling and three of 12 (25\%) patients with STRs. Median PFS was 2.0 versus 3.6 years for patients with STRs versus biopsy/no sampling $(P=\mathrm{ns})$.

\section{Role of histologic subtype}

Sixteen of 20 patients with JPAs progressed, of whom 10 were NED, one AWD, and five were dead of disease at last follow-up. Seven of nine patients with WHO grade II tumors progressed, of whom one was NED, one AWD, and five were dead of disease at last follow-up. For patients with JPAs and WHO grade II tumors, the 15-year PFS was 18.8 and $22.2 \%(P=0.95)$ and 15 -year OS was 73.7 and $55.6 \%(P=0.17)$, respectively. Overall, no statistically significant difference was seen in PFS or OS between grade I and II tumors.

Other factors including KPS, location, sex, and neurofibromatosis were not found to be statistically associated with survival or relapse.

\section{Discussion}

Radiation historically had been a mainstay of treatment for pediatric hypothalamic/chiasmatic LGGs. However, given neurocognitive, endocrine, secondary malignancy, and other long-term consequences, there has been a movement towards attempting to treat children with upfront chemotherapy thus deferring or avoiding radiation $[2,3]$. Various systemic chemotherapy regimens have been studied by investigators with the goal of potentially delaying and perhaps obviating the need for radiation. In this manuscript we report long-term results for 33 patients with hypothalamic/chiasmatic LGGs treated on a phase II TPDCV chemotherapy study. To our knowledge, this is the longest follow-up available in the literature of a phase II chemotherapy trial for pediatric patients with hypothalamic/chiasmatic tumors.

One of the earliest series of chemotherapy for the management of pediatric low-grade gliomas was published by Packer et al. and reported results of using concurrent carboplatin and vincristine. PFS rates were $75 \%$ at 2 years and $68 \%$ at 3 years $[8,9]$.

During this same time period UCSF initiated the study described herein using TPDCV. The original publication focused on chiasmal/hypothalamic gliomas and enrolled 19 children ranging in age from 15 weeks to 15.6 years (median 3.2 years) [6]. At a median follow-up of 79 weeks, median time to tumor progression had not been reached and there were no tumor-related deaths. The four patients who failed chemotherapy were treated satisfactorily with radiation. Of the 18 evaluable patients, 16 had initial improvement of stabilization of visual function.

UCSF investigators reported on an expanded cohort of LGG patients, not limited to OPGs, treated with TPDCV. Of the 42 children in this study, only eight had died, with an estimated 5-year survival of 78\%. Twenty-six patients experienced tumor progression and median time to treatment failure was 132 weeks [5].

The long-term results for 33 patients with chiasmal/ hypothalamic gliomas treated to date on this Phase II study are reported herein. Our study shows favorable OS results, with a 5-year OS of $90.9 \%$ and 15 -year OS of $71.2 \%$ indicating that salvage therapy consisting of radiation, surgery, and/or chemotherapy is successful in many patients.

Our 5-year PFS is $30.3 \%$ and 15 -year PFS is $23.4 \%$. Most patients ( 24 of 25 patients who progressed) had their first progression event within 6 years of diagnosis. Others have found relapses commonly within the first 5 years after diagnosis as well [10]. Salvage therapy varied greatly and included single or combined modality therapy with RT, chemotherapy, and/or surgery. For most young patients, salvage radiation could be delayed until after the age of three.

However, younger patients had much poorer prognosis and many could not be successfully salvaged. All 10 patients that died in our cohort were 3 years old or younger at diagnosis. Younger age was significantly associated with poorer OS and PFS $(P=0.037, P=0.004$, respectively). Of the 18 children who were 3 years or younger at diagnosis, 17 progressed and of these, 10 died. Comparatively, of 15 children who were older than 3 years at diagnosis, eight progressed and none died. Similarly, young age has been found to be associated with poorer outcome by many authors, including four prospective and two retrospective studies [11].

In our study, extent of resection and histology showed no statistically significant benefit in terms of PFS or OS. Based on our data, combined with the majority of published literature, there is no strong evidence that greater extent of resection, without other purpose such as relief of clinical symptoms, is indicated [11].

The regimen reported in this study constitutes one arm of a large scale, randomized phase III, Children's Oncology Group study (COG A9952) examining the relative effectiveness of carboplatin and vincristine (CV) versus TPCV for children $<10$ years of age with progressive or symptomatic unresectable LGGs. Tumor response rates for non-NF1 associated tumors were $57 \%$ for $\mathrm{CV}$ and $58 \%$ for 
TPCV and median time to progression was 3.2 and 4.9 years for the two regimens, respectively [4]. Five-year EFS in non-NF1 randomized subjects was $35 \pm 4.8 \%$ for $\mathrm{CV}$ versus $48 \pm 4.8 \%$ for TPCV $(P=0.11)$. Five-year OS for children with non-NF1 associated tumors was $86 \pm 2.4 \%$ and there were no treatment-related deaths or second malignant neoplasms [4]. These results are comparable to those reported herein, although with 15 year follow up, it is apparent that the vast majority of children experience disease progression and require salvage therapy, most commonly including radiation therapy. A phase II trial (ACNS0223) studied a similar patient subset as A9952 with the addition of temozolomide to $\mathrm{CV}$; the results of this study are pending.

As chemotherapy is increasingly administered in an effort to delay or avoid radiation we must bear in mind that radiation utilized in our study cohort is outdated in many cases and more conformal therapies are now available. In the currently open Phase II COG trial ACNS0221 children aged 3-21 years old with LGGs who have progression or less than a GTR are treated with conformal radiotherapy (3-D, intensity modulated therapy, protons, or stereotactic radiosurgery) to a dose of $54 \mathrm{~Gy}$. This study also has a quality of life component and may eventually provide better guidance as to the optimal use of modern radiation treatment as part of LGG therapy.

Although TPDCV chemotherapy effectively delayed the need for potentially morbid interventions such as surgery and radiation, the long-term PFS of only $23.4 \%$ highlights the need for systemic agents that can be administered chronically with limited side-effects. Molecularly targeted agents offer such promise and have now entered clinical practice. For example, recent studies have shown that aberrant activation of the mitogen-activated protein kinase (MAPK) pathway due to gene duplication or activating mutation of BRAF are a common event in the tumorigenesis of pediatric low-grade astrocytomas. Implication of this signaling cascade in the molecular pathogenesis of pediatric LGGs has opened new avenues for treatment and inhibitors of the MAPK pathway are being proposed for this patient population [12-14]. Similarly, laboratory evidence demonstrating overexpression of vascular endothelial growth factor receptor (VEGF) in pediatric LGGs has led to the use of VEGF inhibitors with promising preliminary results in multiply recurrent pediatric LGGs [15].

In this age of many potential systemic agents, some more toxic than others, it should be noted that for patients who are asymptomatic at presentation, one may consider observation with regular scans, reserving intervention for those who develop clinical signs or symptoms or evidence of progression. Conversely, patients presenting with acute symptoms may require immediate debulking and/or radiation.
In conclusion, long-term outcomes for children with hypothalamic/chiasmatic LGGs treated with upfront TPDCV chemotherapy on a UCSF-BTRC phase II protocol show a survival rate of $71.2 \%$ at 15 -years follow-up. Most patients who will progress do so within the first six years after diagnosis. Young children present a great clinical challenge as their survival and progression prognosis are poor. All 10 children who died in our cohort were 3 years or younger at diagnosis.

Acknowledgments This research was supported in part by NIHPO1 NS-42927-27A2 (D. A. H.-K., K. R. L.), NIH Brain Tumor SPORE grant P50 CA097257 (D. A. H.-K., K. R. L.), The Nancy and Stephen Grand Philanthropic Fund (D. A. H.-K.), and The V Foundation for Cancer Research (DHK), and CA 82103.

Open Access This article is distributed under the terms of the Creative Commons Attribution Noncommercial License which permits any noncommercial use, distribution, and reproduction in any medium, provided the original author(s) and source are credited.

\section{References}

1. Stieber VW (2008) Radiation therapy for visual pathway tumors. J Neuroophthalmol 28:222-230

2. Jahraus CD, Tarbell NJ (2006) Optic pathway gliomas. Pediatr Blood Cancer 46:586-596

3. Massimi L, Tufo T, Di Rocco C (2007) Management of optichypothalamic gliomas in children: still a challenging problem. Expert Rev Anticancer Ther 7:1591-1610

4. Ater A, Holmes E, Zhou T et al (2008) Abstract LGG 18: results of COG protocol A9952: a randomized phase 3 study of two chemotherapy regimens for incompletely resected low-grade glioma in young children. Neuro-Oncology 10:369-537

5. Prados MD, Edwards MS, Rabbitt J et al (1997) Treatment of pediatric low-grade gliomas with a nitrosourea-based multiagent chemotherapy regimen. J Neurooncol 32:235-241

6. Petronio J, Edwards MS, Prados M et al (1991) Management of chiasmal and hypothalamic gliomas of infancy and childhood with chemotherapy. J Neurosurg 74:701-708

7. Kaplan EL, Meier P (1958) Nonparametric estimation from incomplete observations. J Am Stat Assoc 53:457-481

8. Packer RJ, Lange B, Ater J et al (1993) Carboplatin and vincristine for recurrent and newly diagnosed low-grade gliomas of childhood. J Clin Oncol 11:850-856

9. Packer RJ, Ater J, Allen J et al (1997) Carboplatin and vincristine chemotherapy for children with newly diagnosed progressive low-grade gliomas. J Neurosurg 86:747-754

10. Wong JY, Uhl V, Wara WM, Sheline GE (1987) Optic gliomas. A reanalysis of the University of California, San Francisco experience. Cancer 60:1847-1855

11. Opocher E, Kremer LC, Da Dalt L et al (2006) Prognostic factors for progression of childhood optic pathway glioma: a systematic review. Eur J Cancer 42:1807-1816

12. Pfister S, Janzarik WG, Remke M et al (2008) BRAF gene duplication constitutes a mechanism of MAPK pathway activation in low-grade astrocytomas. J Clin Invest 118:1739-1749

13. Jones DT, Kocialkowski S, Liu L et al (2008) Tandem duplication producing a novel oncogenic BRAF fusion gene defines the majority of pilocytic astrocytomas. Cancer Res 68:8673-8677 
14. Jones DT, Kocialkowski S, Liu L et al (2009) Oncogenic RAF1 rearrangement and a novel BRAF mutation as alternatives to KIAA1549:BRAF fusion in activating the MAPK pathway in pilocytic astrocytoma. Oncogene 28:2119-2123
15. Gesundheit B, Klement G, Senger C et al (2003) Differences in vasculature between pilocytic and anaplastic astrocytomas of childhood. Med Pediatr Oncol 41:516-526 